\title{
Langmuir
}

pubs.acs.org/Langmuir

(C) 2009 American Chemical Society

\section{Analysis of Microporosity in Ordered Mesoporous Hierarchically Structured Silica by Combining Physisorption With in Situ Small-Angle Scattering (SAXS and SANS)}

\author{
Simone Mascotto, ${ }^{\dagger}$ Dirk Wallacher, ${ }^{\ddagger}$ Astrid Brandt, ${ }^{\ddagger}$ Thomas Hauss, ${ }^{\ddagger},{ }^{*}$ Matthias Thommes,${ }^{\S}$ \\ Gerald A. Zickler," Sérgio S. Funari, ${ }^{\perp}$ Andreas Timmann, ${ }^{\perp}$ and Bernd M. Smarsly* ${ }^{\dagger}$ \\ $\dagger$ Institute of Physical Chemistry, University of Giessen, Heinrich-Buff-Ring 58, D-35392 Giessen, Germany, \\ ${ }^{\sharp}$ Helmholtz-Zentrum Berlin, BENSC, Glienicker Strasse 100, D-14109 Berlin, Germany, ${ }^{\#}$ Physical \\ Biochemistry, Department of Chemistry, Technische Universität Darmstadt, Petersenstrasse 22, 64287 \\ Darmstadt, Germany, ${ }^{\S}$ Quantachrome Instruments, 1900 Corporate Drive, Boynton Beach, Florida 33426, \\ "Christian Doppler Laboratory for Early Stages of Precipitation, Department of Physical Metallurgy and \\ Materials Testing, University of Leoben, Franz-Josef-Strasse 18, A-8700 Leoben, Austria, and ${ }^{\perp}$ Hamburger \\ Synchrotronstrahlungslabor (HASYLAB)/Deutsches Elektronen-Synchrotron (DESY), Notkestrasse 85, \\ D-22603 Hamburg, Germany
}

Received April 17, 2009. Revised Manuscript Received September 7, 2009

\begin{abstract}
The combination of physisorption experiments with simultaneous in situ small-angle X-ray and neutron scattering (SAXS/SANS) was used to elucidate the porosity in mesoporous silica with a trimodal pore structure. The material ("KLE-IL") contains spherical mesopores of $14 \mathrm{~nm}$ in diameter, worm-like mesopores $(2-3 \mathrm{~nm})$, and micropores, templated by a block copolymer and an ionic liquid surfactant, while the micropores originate from the hydrophilic block of the block copolymer. The main objective of the study was the quantification of the microporosity and the small mesopores and to find out if they are indeed located between the larger, spherical mesopores. Our in situ SAXS/SANS experiments took advantage of contrast matching of nitrogen (SANS, $T=77 \mathrm{~K}$ ) and dibromomethane (SAXS, $T=290 \mathrm{~K}$ ). By using the latter gas with a slightly larger kinetic diameter, it was possible to judge the accessibility of the pores under ambient conditions. The in situ experiments were supported by high-precision ex situ physisorption. Using suitable approaches for the SAXS/SANS analysis, it was possible to separate the content of the micropores and small mesopores.
\end{abstract}

\section{Introduction}

A comprehensive structural characterization of novel nanoporous materials has become more important than ever for the optimization of existing and potentially new applications in the areas of separation, catalysis, gas storage, and so forth. The most popular method to obtain surface area, average pore size, pore size distribution, and porosity information from powders and porous solids is gas adsorption. Significant progress has been achieved during recent years with regard to the understanding of the adsorption mechanism of fluids in narrow pores and the subsequent improvements in the pore size analysis of materials with highly ordered pore structures (e.g., M41S materials). However, there are still many open questions concerning the phase and sorption behavior of fluids in more complex pore systems, such as materials with hierarchical pore structures, which are of interest for practical applications in catalysis, separation, and adsorption. For instance, fluids adsorbed in ordered cage-like mesoporous materials, and hierarchically structured micromesoporous materials can exhibit very complex, but very interesting pore condensation and hysteresis behavior. A combination of phenomena such as delayed pore condensation, pore blocking/percolation,

*Corresponding author. bernd.smarsly@phys.chemie.uni-giessen.de. Tel. +4964199 34590; Fax + 496419934509.

(1) Sarkisov, L.; Monson, P. A. Langmuir 2001, 17, 7600-7604

(2) (a) Ravikovitch, P. I.; Neimark, A. V. Langmuir 2002, 18, 1550-1560. (b) Ravikovitch, P. I.; Neimark, A. V. Langmuir 2002, 18, 9830-9837.

(3) Schreiber, A.; Reinhardt, S.; Findenegg, G. H. Stud. Surf. Sci. Catal. 2002, $144,177-184$.

(4) Vishnyakov, A.; Neimark, A. V. Langmuir 2003, 19, 3240-3247.

(5) Thommes, M. In Nanoporous Materials: Science and Engineering; Lu, G. Q.; Zhao, X. S., Eds.; Imperial College Press: London, 2004; pp 317-364. and cavitation induced evaporation has been observed and discussed in the past years. ${ }^{1-7}$ These complex hysteresis loops introduce of course a considerable complication for pore size analysis, but if interpreted correctly, they provide important information about the pore structure/network, which is crucial for obtaining a comprehensive and accurate textural analysis of advanced micromesoporous materials.

Recently, the combination of a physisorption experiment with small-angle neutron and X-ray scattering (in situ-SANS/SAXSphysisorption) was introduced to provide more details about porosity and connectivity, which cannot be obtained by the single methods themselves. The use of this combined technique gave relevant results on sorption theory and represents an elegant way to describe the physisorption process in mesoporous materials. ${ }^{8-14}$ In particular, it revealed important insights in the study of connectivity and pore accessibility in hierarchical mesoporous frameworks. ${ }^{8,9}$

(6) Libby, B.; Monson, V. Langmuir 2004, 20, 4289-4294.

(7) Kleitz, F.; Czuryszkiewicz, T.; Solovyov, L. A.; Linden, M. Chem. Mater. 2006, 18, 5070-5079.

(8) Sel, O.; Brandt, A.; Wallacher, D.; Thommes, M.; Smarsly, B. Langmuir 2007, 23, 4724-4727.

(9) Smarsly, B.; Göltner, C.; Antonietti, M.; Ruland, W.; Hoinkis, E. J. Phys. Chem. B 2001, 105, 831-840.

(10) Zickler, G. A.; Jähnert, S.; Funari, S. S.; Findenegg, G. H.; Paris, O. J. Appl. Crystallogr. 2007, 40, s522-s526.

(11) Zickler, G. A.; Jähnert, S.; Wagermaier, W.; Funari, S. S.; Findenegg, G. H.; Paris, O. Phys. Rev. B 2006, 73, 184109.

(12) Hofmann, T.; Wallacher, D.; Huber, P.; Birringer, R.; Knorr, K.; Schreiber, A.; Findenegg, G. H. Phys. Rev. B 2005, 72, 064122.

(13) Hoinkis, E. Langmuir 1996, 12, 4299-4302.

(14) Smarsly, B.; Thommes, M.; Ravikovitch, P. I.; Neimark, A. V. Adsorption 2005, 11, 653-655. 
Scheme 1. Structural Constitution of KLE-IL Silica Illustrating the Different Pore Types and Their Filling during Increase in Relative Pressure $^{a}$

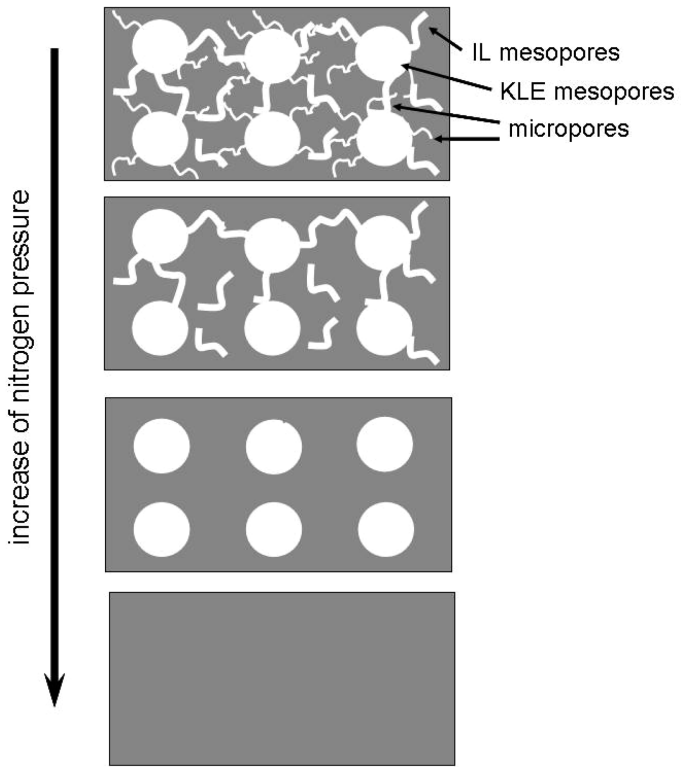

${ }^{a}$ Note that the idealized structural illustration in the upper image, with the micropores and the IL mesopores being homogeneously distributed in the material, was to be proven in the present study.

In our previous work, a hierarchical mesoporous silica was studied in detail, denoted as "KLE-IL". 8 This system reveals a trimodal pore size distribution with maxima at around $1.3 \mathrm{~nm}$, 2-3 nm (cylindrical/worm-like mesopores), and $14 \mathrm{~nm}$ (spherical mesopores). Here, we define a "hierarchical" pore system as a pore architecture with the small pores being located in the walls of the larger ones. In addition to the ordered mesoporosity arising from the templating action of the KLE block copolymer (producing the $14 \mathrm{~nm}$ spherical mesopores) and of the surfactant-like $\mathrm{C}_{16} \mathrm{mimCl}$ ionic liquid (IL) (generating the $2-3 \mathrm{~nm}$ cylindrical/worm-like mesopores), a considerable amount of microporosity is present in such materials. Thus, the pore structure of KLE-IL is considered to be hierarchical, since the micropores $(1.3 \mathrm{~nm})$ and the small mesopores are supposed to be situated between the $14 \mathrm{~nm}$ mesopores (see Scheme 1). A large part of the microporosity is related to the templating of lyotropic phases of block copolymers containing poly(ethylene oxide) (PEO) chains as the hydrophilic block, which is embedded molecularly in the silica matrix, resulting in cavities of similar size in the pore walls after removal of the template by heat treatments. $^{9,15,16}$

The present study represents an extension of our two recent publications on KLE-IL silica, ${ }^{8,17}$ in which the following results were obtained:

1 Ref 17 (2006): It was shown and quantified by high precision nitrogen and argon physisorption measurements that this material possesses 14 nm KLE mesopores, IL mesopores of ca. 2-3, nm and micropores. As main result, it was demonstrated indirectly that emptying of the KLE mesopores is hindered, because they are connected by the smaller pores, and that the

(15) Kruk, M.; Jaroniec, M.; Ko, C. H.; Ryoo, R. Chem. Mater. 2000, 12, 19611968.

(16) De Paul, S. M.; Zwanziger, J. W.; Ulrich, R.; Wiesner, U.; Spiess, H. W. J. Am. Chem. Soc. 1999, 121, 5727-5736.

(17) Thommes, M.; Smarsly, B.; Groenewolt, M.; Ravikovitch, P. I.; Neimark, A. V. Langmuir 2006, 22, 756-764. emptying occurs via cavitation and not pore blocking. Furthermore, the size of the KLE mesopores was confirmed by SAXS measurements.

2 Ref 8 (2007): In this publication, we showed that the in situ SANS/physisorption setup at Helmholtz-Zentrum Berlin (HZB) (formerly Hahn-Meitner-Institute) is suitable to study details of the physisorption in KLEIL silica, using the principle of contrast matching between condensed nitrogen and silica. The in situ SANS data indicated that the majority of the IL mesopores and micropores were located between the KLE mesopores. Thus, the latter result was important with respect to the proof the cavitation mechanism in our former study, as the draining of KLE mesopores through the significantly smaller necks is the origin of the hindered pore emptying.

The current study represents the continuation of these investigations to address further fine details of the porosity and the analysis of mesoporous materials in general. In light of our two previous publications, KLE-IL has turned out to be ideal for our studies on the validation of methods of analyses and on hindered mesopore emptying, because the pore sizes and morphology are exactly known. On the basis of our previous publications, the present study is focused on several detailed issues, which had not been considered in our previous works:

1 Even though in literature can be found several works which address the issue of the microporosity in templated mesoporous systems, ${ }^{18,19}$ this topic in KLE-IL silica, and in similar hierarchical mesoporous systems in general, has not been studied in depth using in situ sorption-SAS methods. Hence, we addressed the question regarding whether it is generally possible to detect micropores in a material with different pore sizes by our in situ scattering setups coupled with physisorption devices. This issue was addressed using the KLE-IL silica as test material.

2 Our previous studies could not reveal whether the micropores are all located between KLE mesopores. As introduced in ref 8 , the analysis of the changes in the Bragg reflection intensity of the KLE mesopores upon filling the micropores serves as an appropriate method.

3 The combined sorption-SAS experiment was used to validate state-of-the-art physisorption analysis: we attempted to quantify the microporosity in such a complex pore system (possessing three types of pores in micro/mesopore region), regarding both the micropore volume and the micropore sizes based on the SANS data. ${ }^{17,20}$

In general, the majority of in situ adsorption/SANS or SAXS experiments are based on the principle of "contrast matching" between silica and the condensed liquid. In this study, for SANS and SAXS we used nitrogen and dibromomethane as the adsorptives, respectively. Liquid nitrogen has got almost the same scattering length density of silica regarding neutrons $\left(\rho_{\mathrm{b}, \text { nitrogen }}=\right.$ $3.22 \times 10^{-6} \AA^{-2}, \rho_{\mathrm{b}, \text { silica }}=3.47 \times 10^{-6} \AA^{-2}$ ). Similarly, condensed dibromomethane has almost the same mean electron

(18) Kim, T. W.; Ryoo, R.; Kruk, M.; Gierszal, K. P.; Jaroniec, M.; Kamiya, S.; Terasaki, O. J. Phys. Chem. B 2004, 108, 11480-11489.

(19) Jun, S.; Joo, S. H.; Ryoo, R.; Kruk, M.; Jaroniec, M.; Liu, Z.; Ohsuna, T.; Terasaki, O. J. Am. Chem. Soc. 2000, 122, 10712-10713.

(20) Sel, O.; Kuang, D. B.; Thommes, M.; Smarsly, B. Langmuir 2006, 22, 2311 2322 . 
Table 1. Porosity Parameters of KLE-IL Silica, Obtained Through the NLDFT Model $^{a}$

\begin{tabular}{ll}
\hline \multicolumn{1}{c}{ property } & value \\
\hline BET surface area $\left(\mathrm{m}^{2} / \mathrm{g}\right)$ & 470 \\
NLDFT surface area $\left(\mathrm{m}^{2} / \mathrm{g}\right)$ & 420 \\
cumulative total pore volume $\left(\mathrm{cm}^{3} / \mathrm{g}\right)$ & 0.34 \\
cumulative micropore volume $\left(\mathrm{cm}^{3} / \mathrm{g}\right)$ & 0.03 \\
cumulative small mesopore volume $\left(\mathrm{cm}^{3} / \mathrm{g}\right)$ & 0.18 \\
cumulative KLE mesopore volume $\left(\mathrm{cm}^{3} / \mathrm{g}\right)$ & 0.13
\end{tabular}

${ }^{a}$ The NLDFT analyses were calculated from the adsorption branch of the nitrogen physisorption isotherm at $77 \mathrm{~K}$ by applying the kernel of metastable adsorption isotherm based on spherical/cylindrical pore model for the system nitrogen $(T=77.4 \mathrm{~K})$ /silica. The BET surface area was calculated in the range $0<p / p^{\circ}<0.15$.

density as silica. The following considerations are referred to nitrogen (SANS) but are also valid for dibromomethane (using SAXS). In SAS experiments, scattering curves are obtained as intensity $I(s)$ versus the modulus $s$ of the scattering vector $s=$ $(2 / \lambda) \sin \theta$, with $\lambda$ being the wavelength and $2 \theta$ the scattering angle. Since this scattered intensity $I(s)$ is related to the square of the difference in scattering length densities $\rho_{\mathrm{b}}$ of void and silica/adsorbate, $I(s) \sim\left(\rho_{\mathrm{b}, \text { void }}-\rho_{\mathrm{b} \text {,silica/adsorbate }}\right){ }^{2}$ only unfilled pores contribute to the scattering signal. ${ }^{9-14}$ Scattering curves can thus provide information on the sorption mechanism as well as the pore system.

Since it is difficult to separate the micropores' contribution from the scattering pattern (i.e., in reciprocal space), the SANS curves obtained during nitrogen physisorption were analyzed by the concept of the "chord-length distribution" (CLD, i.e., in real space). Previously it was demonstrated that this transformation gives illustrative insights into structural and topological details of general two-phase systems with sharp or diffuse phase boundaries. ${ }^{9,21-23}$

Thus, the present study complements our previous studies with a detailed analysis of the microporosity and also shows that in situ-SANS/SAXS-physisorption is a suitable tool to get important information also on small pores in a complex pore structure. A further objective is to demonstrate approaches to analyze the in situ-SAXS/SANS-physisorption data of materials without a very high degree of order, but still possessing a well-defined porosity.

The material used in the present study (KLE-IL silica) could not be taken from the same batch as that described in ref 8 , but was prepared accordingly and thus has the same features regarding the porosity (see Table 1).

\section{Experimental Section}

In Situ SANS. The experiment was performed at the membrane diffractomer V1, which is placed in the cold neutron guide $(\lambda=0.6 \mathrm{~nm})$ of the Helmholtz-Zentrum Berlin. This line is particularly suitable for the study since it allows to analyze higher regions of scattering vector and thus to investigate the micropore regions in more detail. By varying the angle of the sample-detector distance, the scattering intensity was collected in the range of $3.34 \times 10^{-3} \mathrm{~nm}^{-1}<s<1.12 \mathrm{~nm}^{-1}\left(0.1^{\circ}<2 \theta<34^{\circ}\right)$. The scattering vector $s$ is defined as $s=(2 / \lambda) \sin \theta$, with $\lambda$ being the wavelength and $2 \theta$ the scattering angle. The relative pressure of the nitrogen $p / p^{\circ}$ (where $p^{\circ}$ is the saturation pressure at $77 \mathrm{~K}$ ) could be controlled during the experiment. Before performing each scattering measurement, the sample material was filled with a certain amount $\left(n / n^{\circ}\right)$ of condensate by using an appropriate gas adsorption sample environment (CGA-PT), which allows a direct in situ measurement of an entire $p V$ isotherm. The KLE-IL silica material was situated in an aluminum cylindrical cell mounted on

(21) Hoinkis, E. Part. Part. Syst. Charact. 2004, 21, 80-100.

(22) Gille, W. Eur. Phys J. B 2000, 17, 371-383.

(23) Eschricht, N.; Hoinkis, E.; Madler, F. Langmuir 2007, 23, 2145-2157. the coldfinger of a closed-cycle refrigerator stabilized at $77.3 \pm$ $0.004 \mathrm{~K}$.

The collected scattering patterns were then analyzed by means of the chord-length distribution method (see Theory section). This approach can be applied just in the case of ideal two-phase systems. In order to respect this condition by subtraction of a constant background, the Porod law was fulfilled $\left(s^{-4}\right.$ asymptote at high values for $s$ ). The experimental uncertainties in the Porod regime can be due to data noise and three-dimensional fluctuations of scattering length density. After this treatment, the corrected SANS data were evaluated by calculating the CLD in a parametrized form. ${ }^{9}$

In Situ SAXS. For the in situ small-angle X-ray scattering (SAXS) measurements in conjunction with sorption of a fluid in the sample, a special custom-made apparatus was designed. For further details of the setup, see refs 10,11. The sample powder was carefully pressed into a stable pellet of $3 \mathrm{~mm}$ diameter and $0.3 \mathrm{~mm}$ thickness. Before starting a sorption experiment, the sorption cell was evacuated at a pressure below $10^{-3}$ mbar at a temperature of $80^{\circ} \mathrm{C}$ for one hour, but the sample had been kept under vacuum at $120{ }^{\circ} \mathrm{C}$ prior to bringing it into the in situ cell. For sorption experiments, the cell was thermostatted at $17^{\circ} \mathrm{C}$ to be the coldest point in the system, while the liquid reservoir stayed at ambient temperature of $25{ }^{\circ} \mathrm{C}$. The liquid adsorptive dibromomethane $\mathrm{CH}_{2} \mathrm{Br}_{2}$ was used as received. The sorption cell was controlled remotely by a custom-written software program, which allowed continuous adsorption and desorption scans.

In the present work, the KLE-IL silica material was used to study sorption of the organic liquid dibromomethane $\mathrm{CH}_{2} \mathrm{Br}_{2}$ in situ. Using this organic compound, the in situ experiment takes advantage of the principle of scattering contrast matching between the condensed liquid and the amorphous silica matrix, which have almost the identical electron densities. Hence, in the course of increasing the relative vapor pressure $p / p^{\circ}$ of $\mathrm{CH}_{2} \mathrm{Br}_{2}$, filled pores no longer contribute to the scattering signal, and thereby, the filling process can be studied in detail.

The SAXS measurements were performed at the beamlines $\mathrm{A} 2^{24}$ and $\mathrm{BW} 4^{25}$ at the Hamburger Synchrotronstrahlungslabor (HASYLAB)/Deutsches Elektronen-Synchrotron (DESY) in Hamburg, Germany. The synchrotron radiation was monochromatized by a double crystal monochromator to an energy $E$ of $8.27 \mathrm{keV}$ and $8.979 \mathrm{keV}\left(\Delta E / E=10^{-3}\right)$, respectively, focused by a single mirror, and the cross section of the beam was defined by aperture slits to $0.5 \times 0.5 \mathrm{~mm}^{2}$ at the sample position. For the detection of the scattered photons, a charge-coupled device (CCD) X-ray area detector (marCCD 165, marUSA, Evanston, IL, USA) with a resolution of $2048 \times 2048$ pixels (pixel size $79.1 \times$ $79.1 \mu \mathrm{m}^{2}$ ) was used. The precise sample-to-detector distance was determined by a calibration with a standard sample of silver behenate. ${ }^{26} \mathrm{~A}$ total range of the scattering vector $s$ of $0.03 \mathrm{~nm}^{-1}<s<0.38 \mathrm{~nm}^{-1}$ was covered. In order to avoid air scattering, a vacuum flight tube was inserted between the sample and the detector. The transmission of the investigated sample at each sorption state was determined in situ by using an ionization chamber before the adsorption cell for monitoring the primary synchrotron X-ray flux and a photodiode mounted in the beamstop for measuring the transmitted photons. An exposure time of $60 \mathrm{~s}$ yielded a scattering pattern with excellent counting statistics. The scattering patterns were corrected for background scattering, electronic noise, transmission, and polarization by using the data reduction software program FIT2D. ${ }^{27}$ All specimens showed

(24) Elsner, G.; Riekel, C.; Zachmann, H. G. Adv. Polym. Sci. 1985, 67, 1-57.

(25) Roth, S. V.; Döhrmann, R.; Dommach, M.; Kuhlmann, M.; Kröger, I.; Gehrke, R.; Walter, H.; Schroer, C.; Lengeler, B.; Müller-Buschbaum, P. Rev. Sci. Instrum. 2006, 77, 085106

(26) Huang, T. C.; Toraya, H.; Blanton, T. N.; Wu, Y. J. Appl. Crystallogr. 1993, 26, 180-184.

(27) Hammersley, A. P.; Svensson, S. O.; Hanfland, M.; Fitch, A. N.; Häusermann, D. High Press. Res. 1996, 14, 235-248. 
isotropic scattering patterns, which were azimuthally averaged for equal radial distances from the central beam.

\section{Theory}

General Concept of the "Chord-Length Distribution" (CLD). Since the application of a specific structural model is not suitable to analyze the microporosity from the SANS data of the present material, the concept of the so-called "chord-length distribution" (CLD) was applied. This concept, previously introduced by Méring and Tchoubar, ${ }^{28}$ is a suitable method for the qualitative and quantitative analyses of SAXS and SANS of twophase systems. The relationship between the CLD $g(r)$ and the autocorrelation function $\gamma(r)$ is given by

$$
g(r)=l_{\mathrm{p}} \gamma^{\prime \prime}(r) \quad r>0
$$

where $l_{\mathrm{p}}$ is the averaged chord-length ("Porod length") of the system, $l_{\mathrm{p}}$ is the first moment of $g(r)$. The CLD represents a quantitative statistical description for the distances connecting two-phase system boundaries. It contains the complete structural information obtainable from non-normalized scattering intensities such as average pore size, prevalent length scale, correlation lengths, and surface area. The specific surface area per volume $S / V$ is directly correlated to the Porod length $l_{\mathrm{p}}$

$$
l_{\mathrm{p}}=4 \varphi(1-\varphi) V / S
$$

where $\varphi$ is the volume fraction of one of the two phases, $l_{\mathrm{p}}$ is the first moment of the CLD.

In the limit of large $s$, the SAS intensity $I(s)$ is related to $l_{\mathrm{p}}$ and the surface $S / V$ by Porod's law (limit of large $s)^{29}$

$$
I(s)_{s \rightarrow \infty}=\frac{\left(\rho_{1}-\rho_{2}\right)^{2} S}{(2 \pi)^{3} V s^{4}}=\frac{8 \pi Q}{l_{\mathrm{p}} s^{4}}
$$

where

$$
Q=V \varphi(1-\varphi)\left(\rho_{1}-\rho_{2}\right)^{2}=4 \pi \int_{0}^{\infty} s^{2} I(s) \mathrm{d} s
$$

is the so-called "Porod invariant".

The Porod length is also related to the averaged chord-length of the solid $\left(l_{\mathrm{b}}\right)$ and the one of pore/voids $\left(l_{\mathrm{v}}\right)$ by

$$
\frac{1}{l_{\mathrm{p}}}=\frac{1}{l_{\mathrm{b}}}+\frac{1}{l_{\mathrm{v}}}=\frac{1}{\varphi l_{\mathrm{b}}}=\frac{1}{(1-\varphi) l_{\mathrm{v}}}
$$

Thus, $l_{\mathrm{p}}$ allows to calculate the average pore size and average wall thickness without assuming any pore morphology. $9,22,23,30$

\section{Results and Discussion}

Summary. In light of the complexity of the analysis of the SAXS and SANS data, the main results are briefly summarized in the following prior to the detailed description in the following chapters:

1 Our in situ setup allowed for realizing sufficiently small pressures for micropore analysis.

2 The micropore size and the size of the small mesopores $(2-3 \mathrm{~nm})$ can be reasonably determined by the concept of the "chord-length distribution" (section 4.3).

(28) Méring, J.; Tchoubar, D. J. Appl. Crystallogr. 1968, 1, 153-165.

(29) Smarsly, B.; Antonietti, M.; Wolff, T. J. Chem. Phys. 2002, 116(6), 26182627.

(30) Porod, G. in Small Angle X-ray Scattering, Glatter, O.; Kratky, O., Eds.; Academic Press: London, 1982; p 17.
3 The volume fraction of micropores and small mesopores $(2-3 \mathrm{~nm})$ can be determined from SANS/SAXS data by analyzing the intensity shifts of the Bragg peak of the ordered, larger $14 \mathrm{~nm}$ mesopores (section 4.4).

4 The volume fraction obtained from in situ SAXS/ SANS for the micropores and small mesopores are in reasonable agreement with the analysis of ex situ highprecision physisorption data by the NLDFT method.

1. Ex Situ Nitrogen Physisorption Data. Even though the sample analyzed could not be taken from the same batch as in our previous work, it showed identical features regarding the mesoporosity. High-precision ex situ nitrogen physisorption measurements were performed to ensure that the porosity of the sample corresponded to the one previously studied. The sorption characteristics are summarized in Table 1. The BET surface area and the overall pore volume were only slightly different compared to our previous study. ${ }^{8}$

The pore size analysis was carried out by applying the non-local density functional theory (NLDFT). ${ }^{17,31}$ Figure 1 presents the isotherm in linear and semilogarithmic coordinates (Figure 1A, B), the pore size distribution (Figure 1C), cumulative pore volume and cumulative surface area (Figure 1D). It should be noted that there is a difference between the small volume of the micropores and the large surface area that they occupy. On the contrary, the big mesopores created by the templating action of the KLE block copolymer presented a relatively larger pore volume and smaller surface area contribution than the other types of pores. Note that a certain difference is observed between the surface area determined from the BET method and the NLDFT approach, which is probably due to the overlap of sorption in the micropores and IL mesopores in the range where the BET model was applied.

2. In Situ SANS-Nitrogen Sorption Data. Figure 2A shows SANS patterns of the material at different relative pressures of nitrogen between $0<p / p^{\circ}<1$, i.e., at representative points on the adsorption branch of the nitrogen isotherm. For the sake of clarity, and considering that our study is focused on the investigation of the micropores and small IL mesopores, the majority of the SANS curves to be analyzed were taken in the low-pressure region, presented in Figure 2B, corresponding to micropore filling, which lays approximately in the range $0<p / p^{\circ}<0.1 .^{32}$

Similar to our previous work (see ref 8 ), the SANS patterns showed interesting features concerning the porosity and the pore filling of the KLE-IL material. At the void state $\left(p / p^{\circ}=0\right)$, the shape of the SANS curve for scattering vectors $s<0.25 \mathrm{~nm}^{-1}$ was mainly determined by the spherical $14 \mathrm{~nm}$ KLE mesopores (in particular, the prominent Bragg reflection at $s=0.05 \mathrm{~nm}^{-1}$ ). The maxima at $s=0.12 \mathrm{~nm}^{-1}$ and $s=0.2 \mathrm{~nm}^{-1}$ are maxima of the form factor of the spherical KLE mesopores. The broad band at $s=0.25-0.3 \mathrm{~nm}^{-1}$ was assigned to the small IL mesopores, and the scattering at $s>0.42 \mathrm{~nm}^{-1}$ was supposed to originate from micropores.

The basis for the analysis described below is the fact that the mutual arrangement of the micro- and mesopores in the silica matrix is reflected by distinct changes in the relative intensity of the different regions within the SANS or SAXS curves as a function of $p / p^{\circ}$. With the pores being consecutively filled, the SANS curve taken at the evacuated state thus underwent characteristic changes at different pressures and in different regions of the scattering vector. Starting from $p / p^{\circ}=0$, the scattering curve

(31) Ravitkovitch, P. I.; Neimark, A. V. Langmuir 2002, 18, 1550-1560.

(32) Sing, K. S. W. Pure Appl. Chem. 1982, 54(11), 2201-2218. 
A

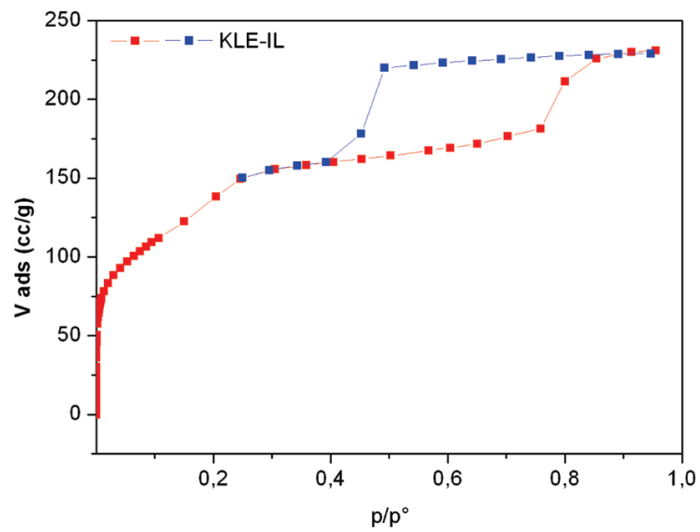

B

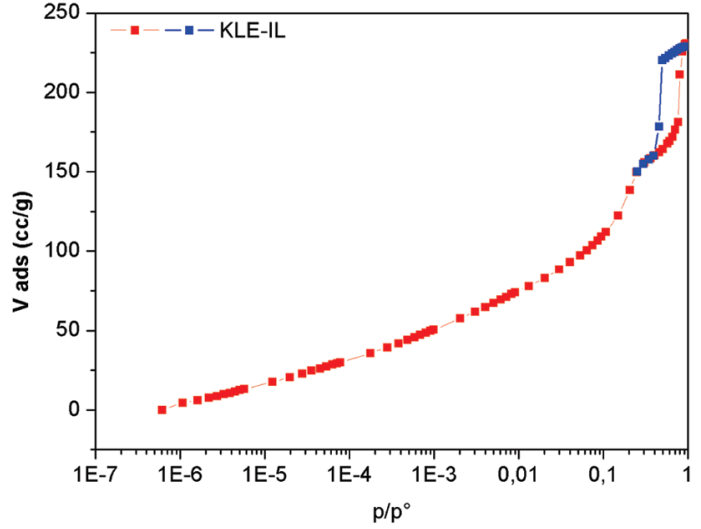

C

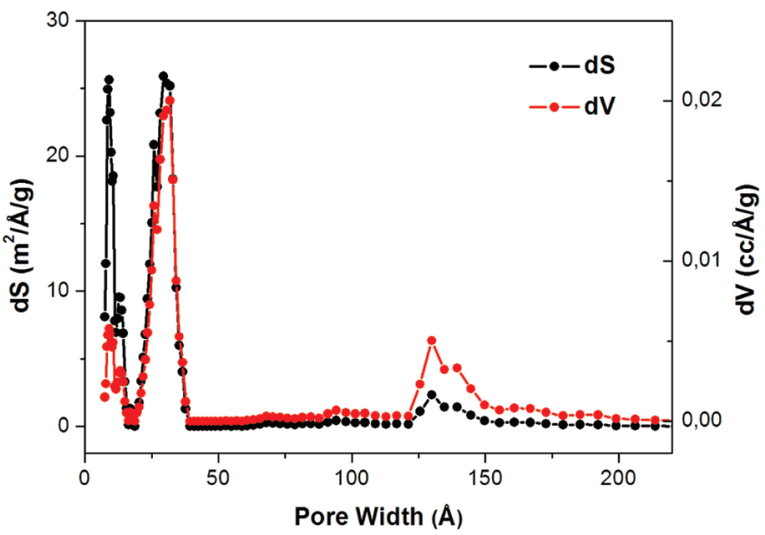

D

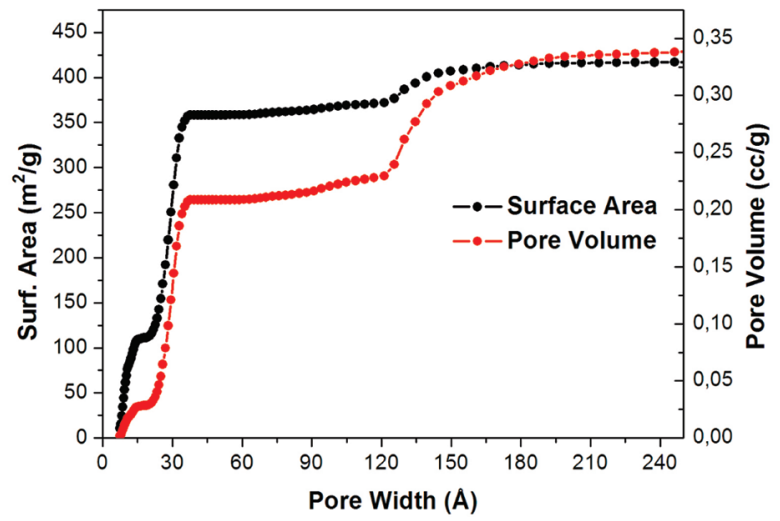

Figure 1. Nitrogen physisorption analysis of KLE-IL silica. (A) Isotherm taken ex situ at $77 \mathrm{~K}$. (B) Semilogarithmic plot of the ex situ isotherm taken at $77 \mathrm{~K}$. (C) Differential pore volume and surface area. (D) Incremental pore volume and surface area. It is important to emphasize that the lines connecting the data points serve just as "guides for the eye" and do not represent any fitting with physical meaning.
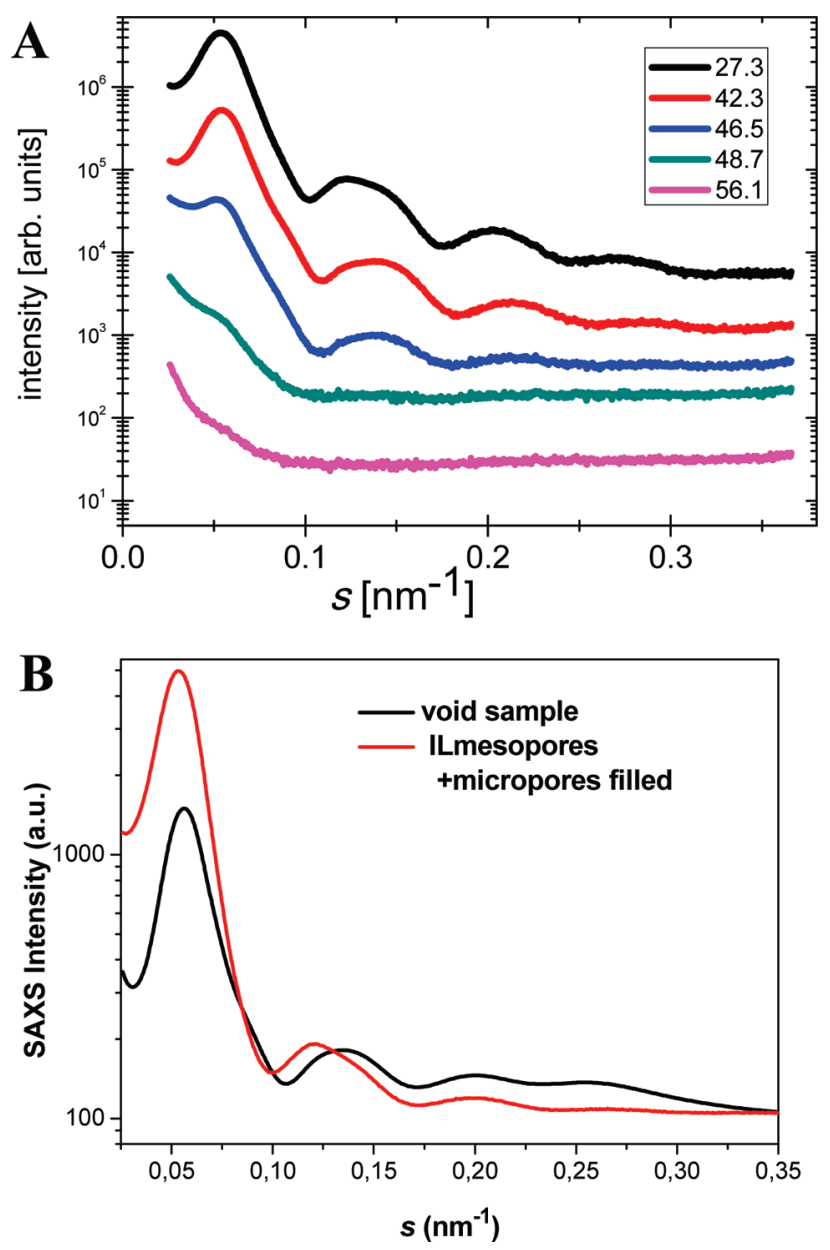

Figure 2. In situ SANS curves of KLE-IL recorded at $0<p / p^{\circ}<1$ (A, overview) and at $0<p / p^{\circ}<0.12(\mathrm{~B})$.

in the region $s<0.1 \mathrm{~nm}^{-1}$ underwent a significant, continuous overall increase up to $p / p^{\circ}=0.2$, which corresponded to the filling of the micropores and small mesopores due to the increase in the average scattering length density of the silica matrix separating the KLE mesopores (see below and ref 8). These findings and the semiquantitative evaluation developed already in ref 8 had provided direct evidence of hierarchical organization of the pore network regarding the IL mesopores and the KLE mesopores, revealing that a vast majority of the IL mesopores were located between the KLE ones. The present set of SANS data thus again confirmed this particular "up" and "down" in the intensity of the first Bragg maximum.

Finally, at $p / p^{\circ}=0.99$ the drastic decrease in the Bragg peak intensity revealed that also the KLE mesopores got filled. The incomplete disappearance of the peak can be referred to the slightly imperfect matching of the scattering length density of silica and nitrogen or to a very small fraction of inaccessible porosity. Note that the SANS data in Figure $3 \mathrm{~A}$ are presented in logarithmic scale, so that the residual intensity appears relatively large, but actually is ca. 1.5 orders of magnitude smaller than in the evacuated state.

3. In Situ SAXS Data Using Dibromomethane. In situ SAXS experiments were performed using dibromomethane as probe gas. In this case, experiments were performed at $T=17^{\circ} \mathrm{C}$, i.e., ambient temperature, using a custom-made sorption cell, combined with a SAXS synchrotron beamline (HASYLAB). The saturation pressure of dibromomethane at this temperature is ca. 58 mbar. Unfortunately, the setup did not allow sufficiently large 

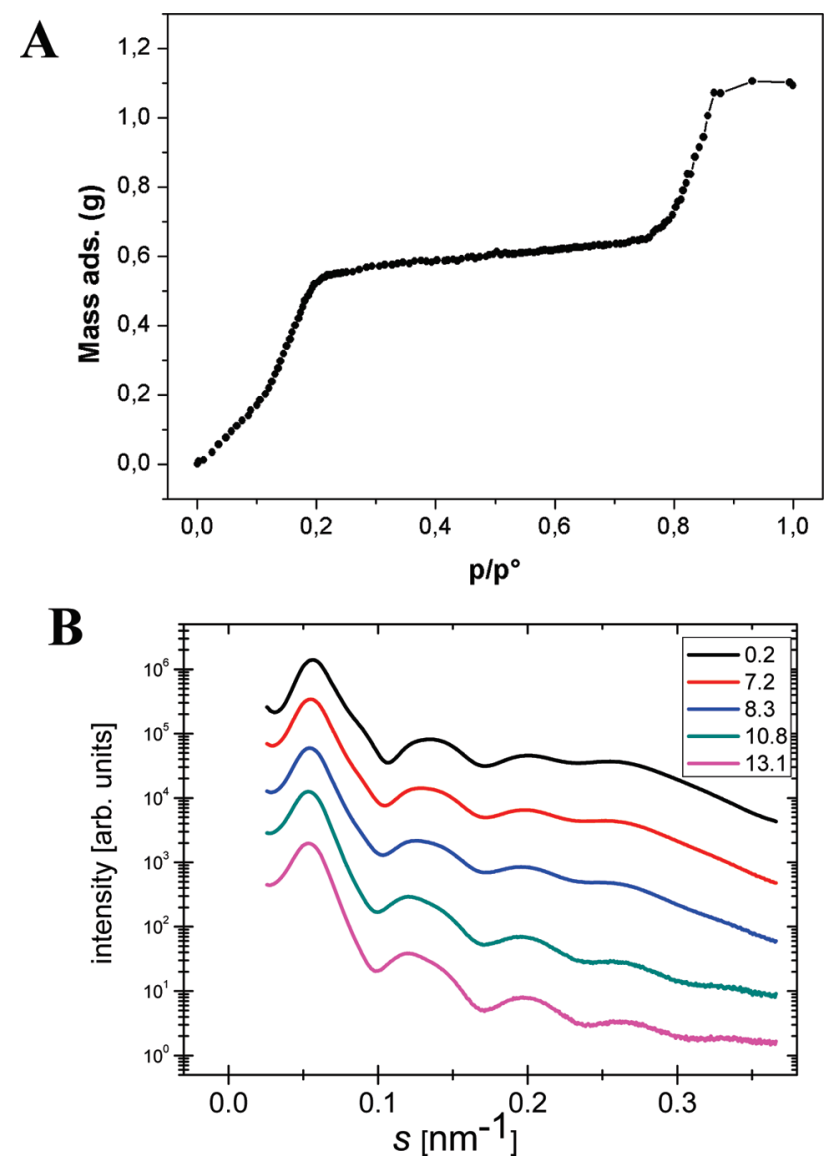

Figure 3. (A) Adsorption isotherm of $\mathrm{CH}_{2} \mathrm{Br}_{2}$ in KLE-IL silica calculated from in situ SAXS transmission measurements. (B) In situ SAXS data obtained during adsorption of dibromomethane. The numbers indicate the absolute pressure in units of mbar. For the sake of clarity, the curves are shifted by a constant factor with respect to each other.

scattering vectors and also very small pressures needed to resolve micropores. Nevertheless, SAXS curves could be accurately measured at pressures where the micropores and IL mesopores were filled (Figure 3B), with the KLE mesopores being empty, thus enabling the quantification of the overall porosity of pores below ca. $3 \mathrm{~nm}$ (see chapter 4.4). It is seen (Figure 3B) that the SAXS curves in the evacuated state were almost identical to those obtained from SANS, thus confirming the usability of the SAXS data. Nevertheless, in the following the SANS data are used, mainly because larger scattering vectors could be more accurately measured than for the SAXS data. As discussed in depth in chapter 4.4, the main Bragg maximum undergoes a significant increase in intensity at pressures where micro- and small mesopores are filled, compared to the evacuated state. Also, the position of the minima shifts corresponding to the form factor of the spherical KLE mesopores. Since the explanation of these changes is quite intricate, it will be dealt with in a separate publication.

4. Analysis of in Situ SANS-Nitrogen Sorption DataFilling of Micro- and Small Mesopores. 4.1. General Aspects of the Chord-Length Distribution (CLD) of KLEIL Silica. Since in our experiment the SANS setup covered a sufficiently large range of the scattering vector with good statistics, the CLD provided a reliable and complete characterization of the entire pore structure, including both micropores and mesopores. As discussed in the following, the CLD itself provided relevant and interesting information on the pore structure of such
A

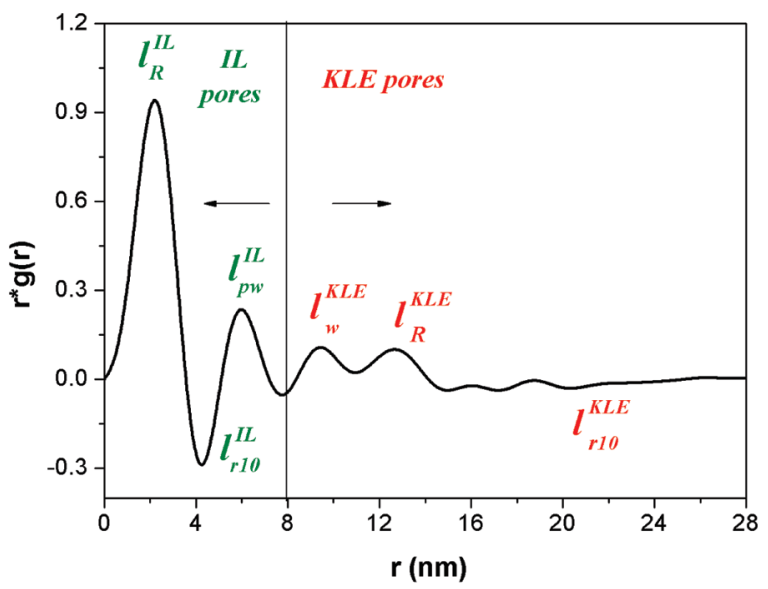

B

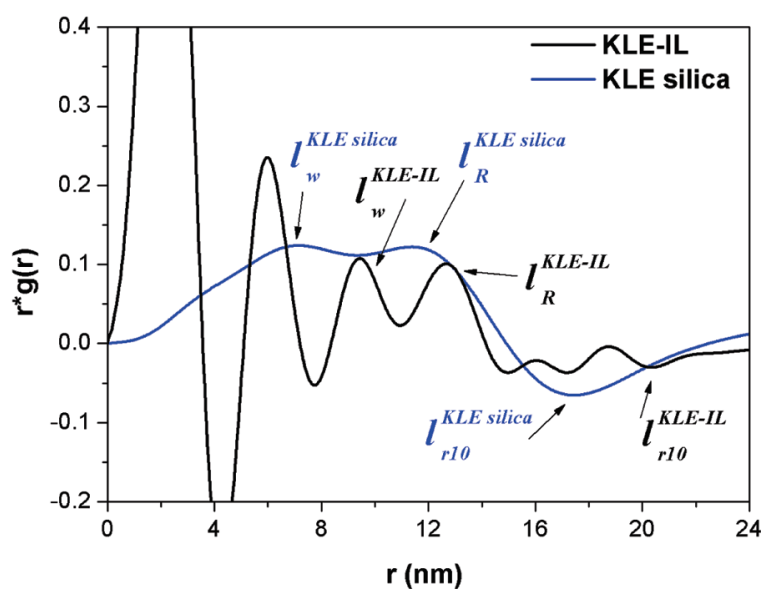

Figure 4. (A) Chord-length distribution (CLD) of KLE-IL silica in the state where the micropores are completely filled $\left(p / p^{\circ}=0.06\right)$ and assignment of the lengths observed in terms of Scheme 2. (B) Comparison of the CLD of KLE-IL silica in the state where the micropores are completely filled $\left(p / p^{\circ}=0.06\right)$ with KLE silica, containing also $14 \mathrm{~nm}$ mesopores and micropores, but no IL mesopores. The KLE silica material was identical to that used in ref 17 (see the references therein concerning the synthesis).

a complex material. The calculation of the CLD from experimental data is delicate, since it is dependent not only of the occurrence of the Porod law, but also on a sufficient data quality at larger scattering vectors $s$. In our experiments, these requirements were met for the SANS data acquired at HZB, allowing us to measure up to $s=1.1 \mathrm{~nm}^{-1}$ with high statistical significance of the data acquired at such large $s$. The Porod plot $\left(s^{4} \times I\right.$ vs $s^{4}$ after background correction, Figure $2 \mathrm{~S}$ in the Supporting Information) reached a plateau with the data points fluctuating moderately around the Porod constant, thus proving sufficient quality of the data at large $s$. For the data analysis, we used a recently described procedure based on an analytical transformation of experimental data. $^{29}$

Figure 4 shows the CLD of KLE-IL silica at $p / p^{\circ}=0.06$, when all the microproes are surely filled, in order to illustrate the shape of the CLD and its interpretation in terms of the void pores (small IL and KLE mesopores) present in the material. In general, the interpretation of CLDs has to be performed with great care, because maxima and minima at certain positions in this distribution do not necessarily directly reflect pores or pore walls of the corresponding size (Scheme 2). In general, the CLD can be regarded as the superposition of the distribution of different kinds of chords connecting different interfaces. For pore morphologies with only local order (as present in our silica), it is legitimate 
Scheme 2. Illustration of the Most Relevant Chord-Lengths Present in a Material Possessing Larger and Smaller Pores

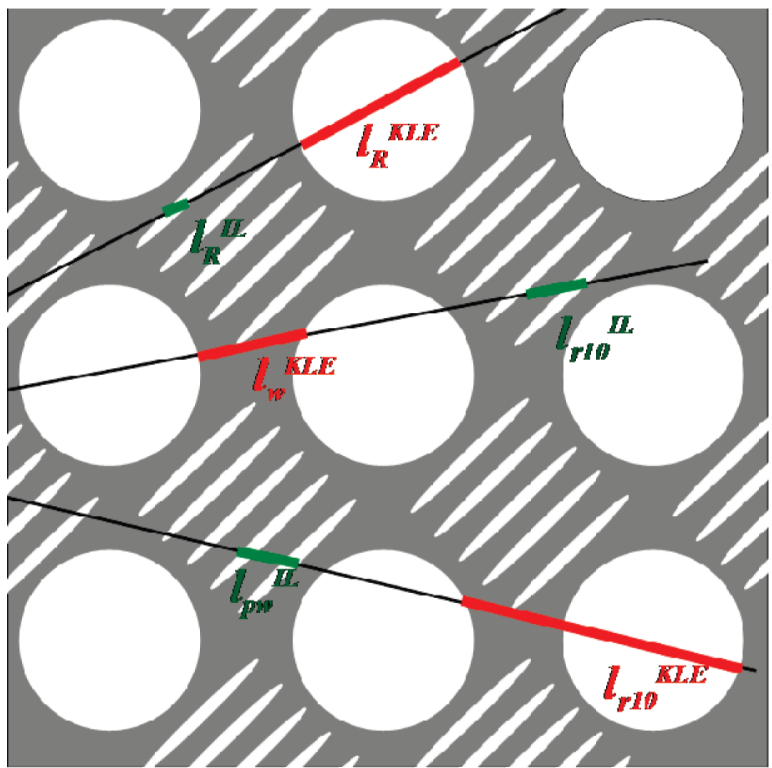

to interpret the first maxima as corresponding to the respective average pore diameters and wall thicknesses. In the case of the present material (Figure 4), the maximum for $0.5 \mathrm{~nm}<r<3 \mathrm{~nm}$ corresponds to the average length of the chords connecting the interfaces of a micro- or IL mesopore. Thus, the minimum of $r$ at about $4 \mathrm{~nm}$ represents the pore-to-pore distance of the IL mesopores. This preferential length corresponds to the maximum seen in the SANS data at $s \approx 0.3 \mathrm{~nm}^{-1}$. Similarly, the maxima at $r=9 \mathrm{~nm}$ and $r=12-13 \mathrm{~nm}$ can be attributed to the walls between the KLE mesopores and the diameter of the KLE mesopores themselves, respectively. This interpretation is strongly supported by the CLD of a silica material only containing KLE mesopores (Figure 4B). ${ }^{17,33}$ The maximum observed at $r=6 \mathrm{~nm}$ probably corresponds to a chord spanning several interfaces (see Scheme 2) and is difficult to assign unambiguously. Such assignment of chords to distinct structural features (pore size, wall thickness, etc.) has to be done with great care and is usually quite speculative. However, as discussed in the following chapter, our assignment and also the conclusions about the microand mesoporosity were supported by the changes seen in the CLDs as a function of the relative pressure.

4.2. Analysis of in Situ SANS-Sorption Data by the CLD Concept. Figure 5 shows the CLDs calculated from the SANS data shown in Figure 2 at various low pressures, i.e., in the interesting region of filling the micro- and small mesopores. Also, in Figure 5A the CLDs are presented up to $r=10 \mathrm{~nm}$ only, thereby focusing on the micropores and small mesopores. Therefore, the impact of the KLE mesopores on the CLDs is not visible (see Supporting Information).

Similar to the SANS data themselves, the CLD has to be interpreted as a superposition of contributions from the three types of pores. Up to pressures of $p / p^{\circ}=0.008$, the CLDs presented a strong contribution at $r<1 \mathrm{~nm}$, which could be attributed to the average size of the micropores, because this signal vanished for $p / p^{\circ} \geq 0.06$, as expected for such small pores. The disappearance of this maximum in the CLD for $p / p^{\circ} \geq 0.06$ coincides with the result from the physisorption analysis (see above). Thus, the filling of the micropores could be monitored

(33) Smarsly, B.; Groenewolt, M.; Antonietti, M. Prog. Colloid Polym. Sci. 2005, 130, 105-113.
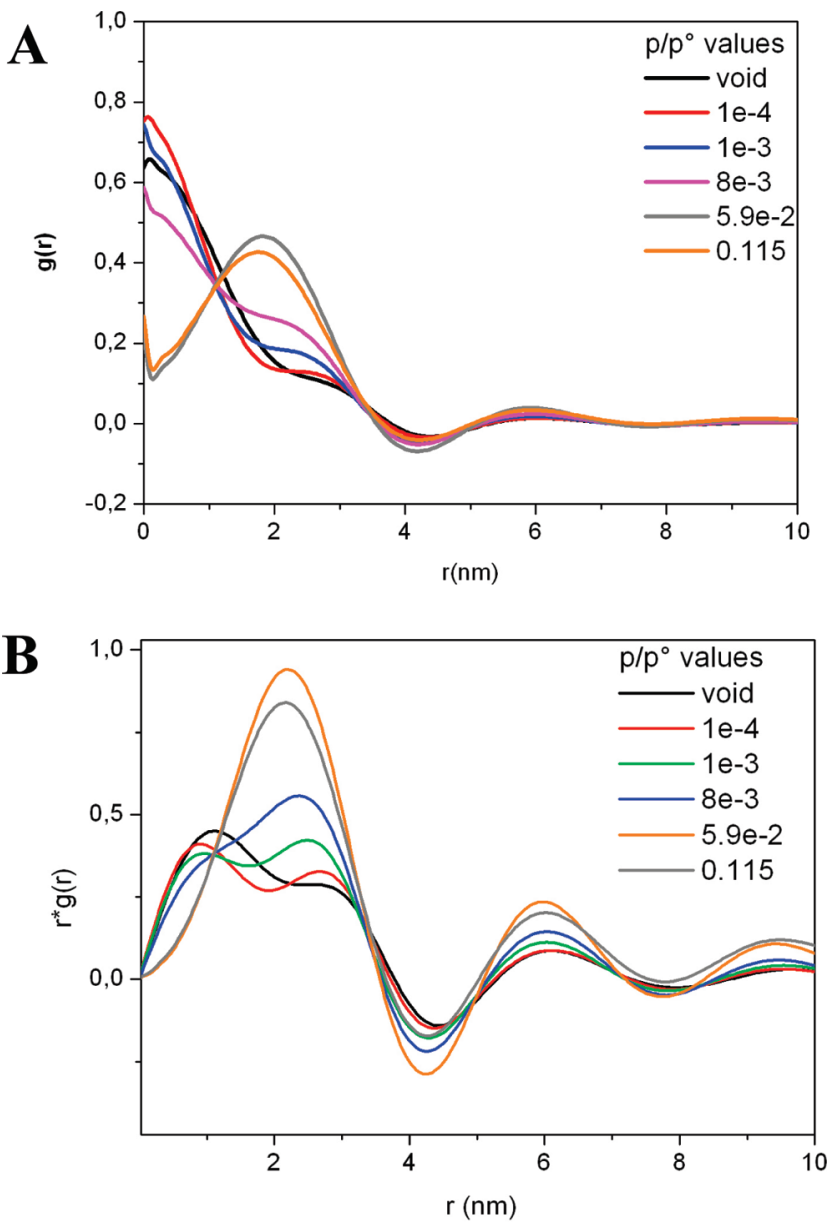

Figure 5. Chord-length distributions $g(r)(\mathrm{A})$ and the representation $r \times g(r)(\mathrm{B})$ calculated from the in situ SANS data shown in Figure 3.

independently by means of the SANS data and their analysis by the CLD concept. In particular, the SANS data were sufficiently accurate and the CLD analysis sufficiently sensitive to prove the absence of unfilled micropores at $p / p^{\circ} \geq 0.06$ implying that indeed the majority of the micropores were accessible. Accordingly, the maximum/shoulder at around $r=2-3 \mathrm{~nm}$ in the CLD curve of the void sample $\left(p / p^{\circ}=0\right)$ corresponded to a superposition of the IL mesopores and the walls separating them. On the basis of the general theory of the CLD, the minimum at $r=4 \mathrm{~nm}$ in this curve can thus be interpreted as the sum of the average IL mesopore diameter and the corresponding pore wall, being in good agreement with an IL mesopore size of $2-3 \mathrm{~nm}$ found by physisorption analysis. Also, this interpretation of the minimum at $r=4 \mathrm{~nm}$ is supported by the fact that it remained at this position with increasing filling, which is expected, since the average poreto-pore distance of the IL mesopores has to stay constant.

This minimum got more distinct at $p / p^{\circ}=0.06$, because the micropores were filled and thus the relative contribution of the IL mesopores to the CLD increased (note that the CLD is a normalized function).

A comprehensive representation of the CLD is the plot of $r \times$ $g(r)$ vs $r$ (Figure 5B), because the prevalent length-scales are obtained from the first momentum of $g(r)$, which is given by $l_{\mathrm{p}}=$ $\int_{0}^{\infty} g(r) \mathrm{d} r$. In particular, assuming that no spatial correlation exists between the micropores and the mesopores, this representation of the CLD of the evacuated material allowed an estimate of the average pore sizes. Under the aforementioned assumption, the 
average micropore size (see Figure 5b and Table 2) was ca. 1$1.2 \mathrm{~nm}$, being in quite good agreement with the NLDFT analysis of ex situ high-precision physisorption data. ${ }^{8,17}$ Note that our experimental SANS setup indeed allowed us to resolve such small pores, because the maximum value of the scattering vector was $s>1 \mathrm{~nm}^{-1}$. Although a truly quantitative interpretation of the CLD has to take into account the pore morphology, evidently good agreement could be obtained in comparison with the physisorption analysis.

Significant changes in both $g(r)$ and $r \times g(r)$ were observed as a function of pressure for $1.5 \mathrm{~nm}<r<5 \mathrm{~nm}$, corresponding to the size range of the IL mesopores. In the CLD itself (Figure 5A) and also in $r \times g(r)$ (Figure 5B), the maximum around $r=2 \mathrm{~nm}$ increased for $p / p^{\circ} \geq 0.008$, appearing as a pronounced maximum for $p / p^{\circ} \geq 0.06$. This increase was due to the definition of the CLD as a normalized distribution: hence, the disappearance of the micropores resulted in a correspondingly higher contribution of the IL mesopores to the CLD. For the same reason, the minimum around $r=4.5 \mathrm{~nm}$ (average distance between mesopores) appeared more pronounced upon an increase in pressure. For the IL mesopores, a maximum (interpreted as a pore size) in $r \times$ $g(r)$ was observed at $r=2.5-3.0 \mathrm{~nm}$, this value being in close agreement with the pore size distribution analysis. However, it should be emphasized that the maximum around $2.5-3.0 \mathrm{~nm}$ also contained the contribution of the silica matrix between the IL mesopores. The $14 \mathrm{~nm}$ KLE mesopores appeared as the contributions beyond ca. $8 \mathrm{~nm}$ and produced the minimum at ca. $r=$ $20 \mathrm{~nm}$, corresponding to the average distance between the KLE mesopores. The layer formation on the walls of the IL mesopores was hardly visible, since the sizes of the IL mesopores and the walls were of the same order of magnitude, thus producing only one maximum in $g(r)$. Also, it should be noted that the elucidation of such fine details exceeds the accuracy of the measurements, taking into account that the IL mesopores possessed significant dispersity in size and shape.

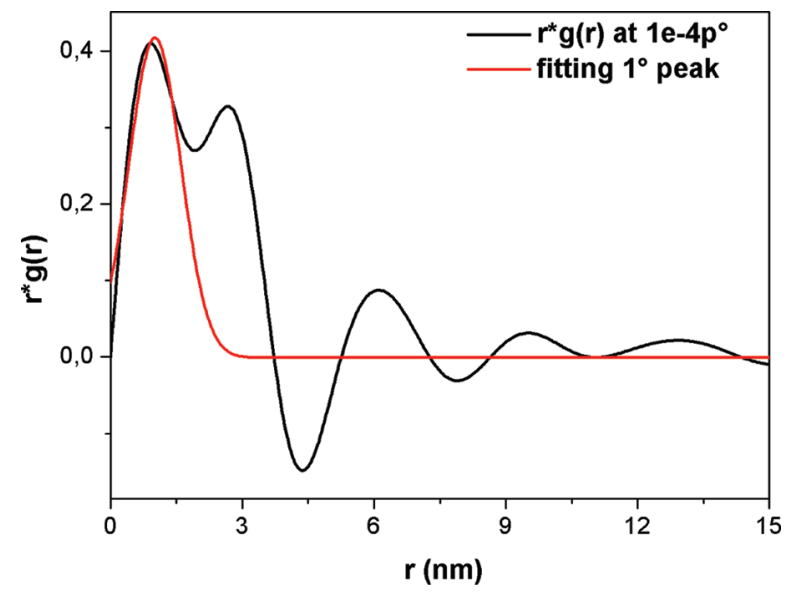

Figure 6. Separation of the micropores in the CLD at $p / p^{\circ}=$ 0.0001 .
In conclusion, the CLD analysis proved to be highly useful to determine the pressure at which filling of micropores and IL mesopores was completed, thus helping to interpret the SANS data themselves. In particular, the increase in the intensity of the Bragg maximum at small pressures (e.g., seen in Figure 2 at $\left.p / p^{\circ}=0.11\right)$ could thus be directly correlated with the "vanishing" (filling) of the micropores seen in the CLD. Thus, this common finding also proves that the majority of the micropores were located between KLE mesopores (see section 4.4).

4.3. Porod Length Analysis. The filling of the micropores and small mesopores was further analyzed by means of the Porod length $l_{\mathrm{p}}$ and related quantities (see above). The $r \times g(r)$ curves, the integrated area of which being $l_{\mathrm{p}}$, were divided into two parts: a first part (see Figure 6) from 0 to $2 \mathrm{~nm}$ representing the micropore region (giving rise to a mean "micropore" Porod length $l_{\mathrm{p} \text {,micro }}$ ), and a second one from 2 to $>20 \mathrm{~nm}$ being mainly determined by the superposition of the small IL mesopores and the walls between them. The influence of the KLE mesopores $(14 \mathrm{~nm})$ can be neglected in such analysis. In this analysis, it was assumed that the micropores and IL mesopores are not spatially correlated; thus

$$
l_{\mathrm{p}} \approx l_{\mathrm{p}, \text { micro }}+l_{\mathrm{p}, \text { ILmeso }}
$$

The obtained values are shown in Table 3 and plotted versus the relative pressure in Figure 7 . Figure $7 \mathrm{~A}$ reveals that $l_{\mathrm{p} \text { micro }}$ possesses a value of ca. $0.7 \mathrm{~nm}$, which can reasonably be interpreted as corresponding to a mean micropore size, considering an experimental error of ca. $10 \%$. Note that for pressures $p / p^{\circ}=0.01$ Figure 7A shows the chord-lengths formally calculated (for the sake of comparability with the lower pressures) as $l_{\mathrm{p} \text {,micro }}$ by the aforementioned method, but these values reflect the chord-lengths of the IL mesopores and the walls separating them, since the micropores are already filled at such pressure. Interestingly, $l_{\mathrm{p}, \text { ILmeso }}$ increased already below $p / p^{\circ}=0.1$. Despite the imprecision in the estimation of $l_{\mathrm{p} \text {,micro }}$ due to the superposition of different sorption phenomena taking place at the same time like micropore filling and sorption in IL mesopores, through eq 1 it is possible to estimate the value of the micropore volume fraction $\left(\tilde{\phi}_{\text {micro }}\right.$, the definition is given in eq 5$)$ of the sample from the CLD of the evacuated sample, i.e., by $l_{\mathrm{p}, \text { micro }}=4 \tilde{\phi}_{\text {micro }}(1-$ $\left.\tilde{\phi}_{\text {micro }}\right) V / S_{\text {micro }}$. While $l_{\text {p,micro }}$ was obtained from in situ SANS (via separation from the CLD; see Figure 7), $S_{\text {micro }} / V$ could be calculated from the physisorption data: In principle, the micropore surface area per volume $S_{\text {micro }} / V$ is given by

$$
\frac{S_{\text {micro }}}{V}=\frac{S_{\text {micro }}}{m} \rho_{\mathrm{SiO}_{2}}\left(1-\tilde{\phi}_{\mathrm{TOT}}\right)
$$

where $\rho_{\mathrm{SiO}_{2}}$ is the mass density of silica, $\tilde{\phi}_{\text {TOT }}$ the total volume fraction of all kinds of pores (calculated from eq 5 using the values obtained by NLDFT model, Table 1 ) and $S_{\text {micro/m }}$, the micropore surface area per gram, also obtained by the sorption measurement. On the basis of the analysis of $l_{\mathrm{p}}$ from the SANS of the evacuated sample, we thus obtain a value for $\tilde{\phi}_{\text {micro }}=0.025$,

\begin{tabular}{|c|c|c|c|}
\hline property & NLDFT & CLD analysis & in situ SANS/SAXS Bragg peak analysis \\
\hline volume fraction of all pores $\left(\tilde{\phi}_{\mathrm{TOT}}\right)$ & 0.42 & n.a.* & 0.41 \\
\hline volume fraction of micopores and IL mesopores $\left(\tilde{\phi}_{\mathrm{IL}}\right.$ mesopores + micropores $)$ & 0.26 & n.a.* & 0.25 \\
\hline volume fraction of IL mesopores $\left(\tilde{\phi}_{\mathrm{IL} \text { mesopores }}\right)$ & 0.22 & n.a.* & 0.18 \\
\hline volume fraction of micropores $\left(\tilde{\phi}_{\text {micronores }}\right)$ & 0.036 & 0.025 & 0.07 \\
\hline micropore relative area & $27 \%$ & $24 \%$ & n.a.* \\
\hline micropore size $(\mathrm{nm})$ & $0.9-1.3$ & $1-1.2$ & n.a.* \\
\hline
\end{tabular}

Table 2. Porosity Analysis of KLE-IL Silica Obtained through NLDFT Method, Chord-length Distribution Approach and Bragg Peak Analysis ${ }^{a}$ 
Table 3. Values of $l_{\mathrm{p} \text {,micro }}$ and $l_{\mathrm{p}, \text { tot }}$ Obtained by the Separated CLD Plots at Different Relative Pressure $p / p^{\circ}$

\begin{tabular}{ccc}
\hline \multicolumn{1}{c}{$p / p^{\circ}$} & $l_{p_{-} \text {micro }}(\mathrm{nm})$ & $l_{p_{-} \text {tot }}(\mathrm{nm})$ \\
\hline $1 \times 10^{-4}$ & 0.6 & 1.0 \\
$1 \times 10^{-3}$ & 0.6 & 1.10 \\
0.008 & 0.8 & 1.30 \\
0.029 & 0.8 & 1.4 \\
0.059 & - & 1.90 \\
0.115 & - & 2.0
\end{tabular}

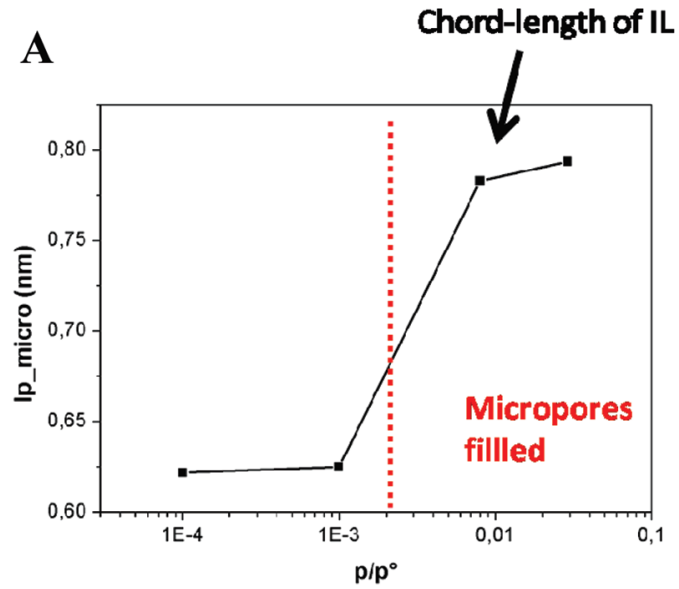

B

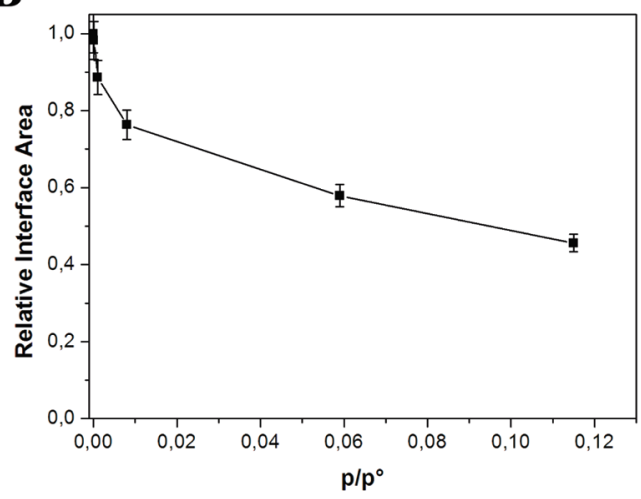

Figure 7. (A) Variation of the average chord-length $l_{\mathrm{p} \text {,micro }}$ of the micropores, obtained via the separation of the CLD. The step increase at $p / p^{\circ}=0.01$ corresponds to structural features larger than the micropores; i.e., micropores are filled at this state. (B) Change of the remaining interfacial area as a function of $p / p^{\circ}$.

which is near the one $\left(\tilde{\phi}_{\text {micro }}=0.036\right)$ calculated by the sorption analysis with the NLDFT kernel (Table 1).

The CLD can also be used to calculate the relative interfacial area as a function of relative pressure. The remaining interface area was calculated from the changes in the Porod invariant $Q$ and $l_{\mathrm{p}}$ as described in eqs $1-3 .{ }^{9,23,34}$ Assuming that for our sample the majority of the micropores were filled at $p / p^{\circ}=0.008$, as described above, we can estimate a free interfacial area of $76 \%$ for this pressure. This value means that the contribution of the micropores to the overall surface area corresponded to $24 \%$. The NLDFT evaluation (Table 1) provided a relative contribution of the micropores to the overall surface of $27 \%$, which is obviously in good agreement with the value calculated through the Porod invariant $Q$. It can be excluded that the drop of surface area is significantly influenced by sorption in the IL mesopores and KLE mesopores, since the CLDs did not reveal drastic

(34) Steriotis, T.; Mitropoulos, A.; Kanellopoulos, N.; Keiderling, U.; Wiedenmann, A. Physica B 1997, 234, 1016-1018. changes at the corresponding dimensions. Figure 7B also nicely qualitatively illustrates the continuous decrease in the surface area caused by layer formation at higher pressures.

4.4. Analysis of the Intensity of the Bragg Reflection as a Function of $p / p^{\circ}$. 4.4.1. General Considerations. The following analysis is based on the procedure introduced in ref 1 . The main goal is to obtain accurate values for the volume fraction of micropores and IL mesopores from the changes in the intensity of the Bragg peak at $s=0.05 \mathrm{~nm}^{-1}$ generated by the well-ordered KLE mesopores. Thus, this analysis allowed direct comparison with physisorption analysis by NLDFT. To determine the micropore filling (and to separate it from the filling of the IL mesopores), one has to focus on the SANS data in the pressure region $0<p / p^{\circ}<0.1$.

It is seen by the CLD analysis (see above) that the micropores started to be filled at quite low pressure, visible at ca. $p / p^{\circ}=10^{-4}$ in our in situ experiments. At $p / p^{\circ}=0.115$, the micropores were certainly completely filled (see CLD analysis), most probably already at $p / p^{\circ}=0.008$. Evidently, the main problem in separating the micropore filling from the adsorption in the IL is the fact that at such small pressures adsorption (layer formation) takes place also in the IL mesopores, while the condensation can be neglected, of course. However, not even one monolayer is formed on the IL and KLE mesopores at such low pressures. Subsequently, at higher $p / p^{\circ}$ also the small IL mesopores began to be filled, as can be seen by the vanishing of the corresponding scattering maximum at around $s=0.3 \mathrm{~nm}^{-1}$ in the SANS data. This series also evidenced the increase of the overall SANS intensity of the Bragg reflection at $s=0.05 \mathrm{~nm}^{-1}$ already at small pressures (Figure 2b). ${ }^{8}$ In particular, the intensity of the Bragg maximum continuously increased during filling of the micro- as well as the IL mesopores. As pointed out recently, ${ }^{8}$ the changing of the relative overall intensity of the SANS curves was due to the increase in the average scattering length density of the silica surrounding the KLE mesopores, owing to the filling of the small IL mesopores and micropores, and thus contained information about the pores' mutual arrangement in the material and the amount of these smaller pores. Similarly, here the enhancement of the Bragg reflection for very low pressures already asserted that the micropores and IL mesopores were placed in between the KLE mesopores for this batch of material also.

In section 4.4.2, we describe the problem of the simultaneous micropore filling and layer formation in IL pores, which impedes the micropore analysis, and summarize the result. In section 4.4.3, the procedure is given in detail.

4.4.2. The Problem of Separating Micropore Filling and Layer Formation on the Walls of the IL and KLE Mesopores. The quantitative procedure described in section 4.4.3 to calculate the micropore and IL mesopore volume from the changes in the Bragg reflection of the KLE mesopore structure is based on the assumption that micropore filling and layer formation on the IL mesopores are subsequent phenomena. However, even in the lowpressure range where the micropores completely fill (at $p / p^{\circ}<$ 0.015 ) with adsorbate, adsorption (i.e., formation of a monolayer) takes place on the pore walls of the IL and KLE mesopores. However, in spite of this effect the determination of microporosity is still possible:

1 This formation of an adsorbed layer does not influence the analysis of the KLE Bragg reflection on the KLE mesopores, because it practically does not affect the mean density of the surrounding silica matrix. More importantly, the maximum thickness at such low pressures is ca. $0.3 \mathrm{~nm}$ (see below), which is 
significantly smaller than the KLE mesopore diameter. Such submonolayer layer formation has no appreciable effect on the form factor of the KLE mesopores (see Supporting Information) at those scattering vectors, where the Bragg reflection appears, i.e., the absolute intensity of the Bragg reflection is hardly affected by this slight modulation of the form factor of KLE mesopores.

2 However, in the case of the narrow IL mesopores (diameter ca. $3 \mathrm{~nm}$ ) the formation of an adsorbed layer on the IL mesopore walls impacts the SAXS/ SANS Bragg analysis presented here. For instance, at $p / p^{\circ}=0.008$ (see Figure $2 \mathrm{~b}$ ), where the majority of micropores has already been filled, the statistical thickness of the adsorbed liquid-like $\mathrm{N}_{2}$ adsorbate layer is ca. $0.3 \mathrm{~nm}$, based on a $\mathrm{N}_{2} 77 \mathrm{~K}$ reference isotherm obtained on amorphous silica LiChrosper Si-1000 silica, ${ }^{35}$ which is close to monolayer thickness. This seems to be a reasonable value, since from an analysis of the $\mathrm{N}_{2}$ isotherm on KLE silica in ref 10 one obtains a statistical thickness of $0.25 \mathrm{~nm}$ at a $p / p^{\circ}=$ 0.008 , if one assumes that based on the BET analysis a monolayer is completed at $p / p^{\circ}=0.1$. Hence, assuming an adsorbed layer thickness of ca. $0.3 \mathrm{~nm}$ (as an upper limit) would result in a situation in which ca. 1/3 of the IL pore volume has already been filled. Despite this obstacle, it is still possible to extract the micropore volume fraction from an analysis of the scattering data: as described in the Supporting Information, the micropore volume is given by the value obtained from the "Bragg analysis" for $p / p^{\circ}=0.008$ (see section 4.4.3) minus the volume occupied by the statistical layer in the IL mesopores. From the Bragg analysis, we obtained $V_{\text {micropores+ILlayer }}=0.07 \mathrm{~cm}^{3} / \mathrm{g}$ (see 4.4.3), while from NLDFT we obtained ca. 0.03 $\mathrm{cm}^{3} / \mathrm{g}$ (see Table 1). The difference $\left(0.04 \mathrm{~cm}^{3} / \mathrm{g}\right)$ is thus in perfect agreement with the volume expected for a statistical layer on the IL pores $\left(0.04 \mathrm{~cm}^{3} / \mathrm{g}\right.$, see Supporting Information).

4.4.3. Determination of Micropore and IL Mesopore Volume from the SAXS/SANS Reflection of the KLE Mesopores. In general, the volume fraction of the micropores relative to the volume of the walls between the KLE mesopores is given by eq $4 \mathrm{c}$. It is important to emphasize that in the following context $\phi_{\text {micropores }}$ (and accordingly $\phi_{\mathrm{IL}}$ mesopores and $\phi_{\mathrm{IL}}$ mesopore+micropores) is defined as volume fraction in relation only to the KLE pore walls, and not to the entire volume (which is the sum of pore volume plus the volume occupied by silica).

$$
\begin{gathered}
\phi_{\text {ILmesopores }}=\frac{V_{\text {ILmesopores }}}{V_{\text {ILmesopores }}+V_{\text {micropores }}+V_{\mathrm{SiO} 2}} \\
\phi_{\text {ILmesopores+micropores }}=\frac{V_{\text {ILmesopores }+ \text { micropores }}}{V_{\text {ILmesopores+micropores }}+V_{\mathrm{SiO} 2}} \\
\phi_{\text {micropores }}=\frac{V_{\text {micropores }}}{V_{\text {ILmesopores }}+V_{\text {micropores }}+V_{\mathrm{SiO} 2}}
\end{gathered}
$$

These $\phi$ values are defined in this way, because it is these quantities which can be obtained from the SAXS/SANS Bragg peak analysis

(35) Jaroniec, M.; Kruk, M.; Olivier, J. P. Langmuir 1999, 15, 5410-5413. (see below). By contrast, by $\tilde{\phi}$ we define the volume fraction of pores, in relation to the entire volume (i.e., including the KLE mesopores)

$$
\widetilde{\phi}_{\text {micropores }}=\frac{V_{\text {micropores }}}{V_{\text {micropores }}+V_{\text {ILmesopores }}+V_{\mathrm{KLEpores}}+V_{\mathrm{SiO} 2}}
$$

$\tilde{\phi}_{\text {IL mesopores }}$ and $\tilde{\phi}_{\text {IL mesopores+micropores }}$ are defined accordingly. These $\tilde{\phi}$ values are easily obtained from physisorption analysis, but cannot be obtained directly from the SANS data.

The procedure to extract the micropore volume fraction differs slightly from the one present in our previous work because the influence of both micro- and IL mesopores on the Bragg peak has to be considered: $\left(1-\phi_{\text {micropores }}\right)=\sqrt{\frac{I_{\mathrm{KLE}-\mathrm{IL}, \text { evacuated }}(s)}{I_{\mathrm{KLE}}-\mathrm{IL} \text {,mircoporesfilled }(s)}}$

$I_{\mathrm{KLE}-\mathrm{IL}, \text { evacuated }}(s)$ and $I_{\mathrm{KLE}-\mathrm{IL}, \text { micropores filled }}(s)$ are the intensities (i.e., maxima) of the Bragg reflection of the KLE mesopores in the void state and for the situation where the micropores are filled, respectively. Note that it is legitimate to use the absolute values of the Bragg maximum and not mandatory to use the integral intensities, because the shape of the Bragg reflection remains unchanged upon the increase. In our recent study, we obtained a volume fraction of $\phi_{\mathrm{IL} \text { mesopores+micropores }}=0.42$ for the sum of the micro- and mesopores in the pore walls between the KLE mesopores.

While we define $\rho_{\mathrm{b}} \mathrm{SiO}_{2}$ as the scattering length density of amorphous silica (according to a mass density of ca. $2.2 \mathrm{~g} / \mathrm{cm}^{3}$ ), we define $\bar{\rho}_{\mathrm{b} \text { evacuated }}$ as the average scattering length density of the silica matrix between the KLE mesopores in the evacuated state. We assume that the scattering length densities of silica and condensed nitrogen are practically identical (which is a valid assumption within an error of maximum ca. $5 \%$ ). Hence, $\bar{\rho}_{\mathrm{b}}$ filled micropores is defined as the scattering length density of the matrix between the KLE mesopores with the micropores being filled but the IL mesopores being empty. Accordingly, $\bar{\rho}_{\mathrm{b}}$ KLE-IL, filled IL is defined as the scattering length density of the silica with IL mesopores (and hence also micropores) being filled. For this state, thus $\bar{\rho}_{\mathrm{b} \text { KLE-IL, filled IL }} \approx \rho_{\mathrm{b}} \mathrm{SiO}_{2}$ holds true.

$$
\begin{aligned}
\rho_{\mathrm{b} \text { evacuated }} & =\rho_{\mathrm{b} \mathrm{SiO}}\left(1-\phi_{\text {ILmesopores }}-\phi_{\text {micropores }}\right) \\
& \approx \rho_{\mathrm{b} \mathrm{SiO}}\left(1-\phi_{\text {ILmesopores }}\right)\left(1-\phi_{\text {micropores }}\right)
\end{aligned}
$$

The latter approximation is valid, because $\phi_{\mathrm{IL} \text { mesopores }} \phi_{\text {micro- }}$ pores is rather small. This "trick" now allows the development of a relationship between the intensity of the Bragg reflection and the scattering length densities, as follows:

$$
\rho_{\mathrm{b} \text { evacuated }}=\rho_{\mathrm{b} \text { filledmicropores }}\left(1-\phi_{\text {micropores }}\right)
$$

$$
\text { with } \quad \rho_{\mathrm{b} \text { filledmicropores }}=\rho_{\mathrm{SiO} 2}\left(1-\phi_{\mathrm{ILmesopores}}\right)
$$

From the increase in intensity of the first Bragg maximum at $p / p^{\circ}$ of ca. 0.01 (filled micropores), we obtain

$$
\frac{I_{\text {evacuated }}}{I_{\text {filled micropores }}}=\frac{\bar{\rho}_{b \text { evacuated }}^{2}}{\bar{\rho}_{b \text { filled micropores }}^{2}}=k \text { and } \frac{\bar{\rho}_{\text {b evacuated }}}{\bar{\rho}_{\text {filled micropores }}}=\sqrt{k}
$$

$$
\text { Consequently, it follows }\left(1-\phi_{\text {micropores }}\right)=\sqrt{k}=\sqrt{\frac{I_{\text {evacuated }}}{I_{\text {filled micropores }}}}
$$

Equation 8 thus allows the calculation of the micropore fraction with the KLE pore walls just from the intensity of the absolute 
Scheme 3. Different Scenarios for the Distribution of the KLE Mesopores (spherical $14 \mathrm{~nm}$ mesopores), IL Mesopores (worm-like, 2-3 nm) and Micropores ${ }^{a}$

A

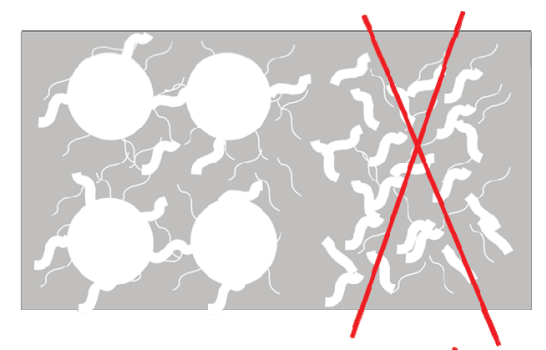

B

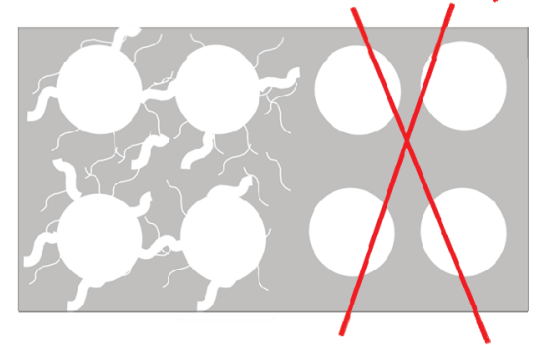

C

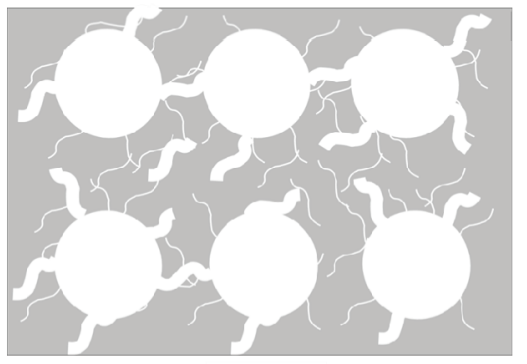

${ }^{a}$ The inhomogeneous pore distributions $\mathrm{A}$ and $\mathrm{B}$ can be excluded by the in situ SAXS/SANS experiments. In contrast, scenario C is valid, i.e., a homogeneous distribution of the small pores situated between the spherical mesopores.

value of the Bragg maximum in the evacuated state and the state with filled micropores. From the SANS data, we obtain $I_{\text {evacuated }}(S) /$ $I_{\text {filledmicropores }}(s)=0.7$, and using eq $8, \phi_{\text {micropores }}=0.16$. Note that this value $\phi_{\text {micropores }}$ represents the volume fraction of micropores in relation to the pore walls separating the KLE pores and not to the entire sample $\left(\tilde{\phi}_{\text {micropores }}\right)$. (Note that the value for the micropore volume fraction is blurred by the presence of layer formation in IL mesopores at small pressures.)

Applying the analysis of the Bragg reflection intensity to the changes in SANS intensity at pressures when the IL mesopores are also filled (at $p / p^{\circ}$ ca. 0.25; see isotherm), we obtain $\phi_{\mathrm{IL} \text { mesopores+micropores }}=0.3$ for the volume fraction of microand IL mesopores in the KLE pore walls (corresponding to an overall volume fraction of micro- and IL mesopores in the sample of $\tilde{\phi}_{\text {IL mesopores+micropores }}=0.25$ ). Thus, the volume fraction of the mesopores $\phi_{\mathrm{IL}}$ mesopores in the pore walls amounts to ca. 0.14 , corresponding to $\tilde{\phi}_{\mathrm{IL}}$ mesopores $=0.18$ being in good agreement with high-precision ex situ physisorption analysis. These values could also be accurately obtained from the in situ SAXS measurements using $\mathrm{CH}_{2} \mathrm{Br}_{2}$; we obtained $\tilde{\phi}_{\mathrm{IL} \text { mesopores+micropores }}=0.26$, i.e., in excellent agreement with the in situ SANS data. In summary, the porosity values obtained from SAXS/SANS are in good agreement with NLDFT physisorption analysis. The only main difference is given in the calculation of the micropore volume fraction. In this case, the value obtained by means of the scattering method could be overestimated, with a consequential underestimation of $\tilde{\phi}_{\mathrm{IL} \text { mesopores. }}$ This effect can be explained by the fact that the filling of the micropores is accompanied by the formation of layers of adsorbate in the small IL mesopores, which were, by definition, assumed empty. The difference between the micropore volumes determined by the analysis in this chapter and NLDFT (see Table 2) can be explained and reasonably estimated on the basis of a straightforward calculation (see section 4.4.2 and Supporting Information).

The value of $\tilde{\phi}_{\text {micropores }}=0.07$ as determined by SANS/SAXS corresponds reasonably to the sum of the micropore volume determined by NLDFT analysis and the volume occupied by a adsorbate on the IL mesopore walls (see section 4.4.2 and Supporting Information). At least $50 \%$ of the value $\tilde{\phi}_{\text {micropores }}=$ 0.07 is attributable to microporosity. The micropore value obtained from the SAXS/SANS Bragg evaluation represents a quite reasonable approximation of the dimension of the micropore volume even in the presence of a significantly larger volume of the IL mesopores. It is important to note that the value of increase in the Bragg intensity at $p / p^{\circ}=0.008$ cannot solely be explained by layer formation in the IL mesopores, but requires the presence of micropores. Thus, the approach of analyzing the SAXS/SANS is in full compliance with physisorption analysis.

\section{Conclusions}

In the present study, in situ SANS and SAXS data were obtained during sorption of nitrogen and dibromomethane, respectively, using a hierarchically structured silica with three types of pores (micropores, 2-3 nm mesopores, $14 \mathrm{~nm}$ ordered spherical mesopores). In particular, it is demonstrated that, using a suitable in situ setup, in situ SANS and SAXS allows the study of not only the mesoporosity, but also the microporosity of complex hierarchical pore structures with sufficiently high accuracy by comparing with state-of-the-art physisorption experiments coupled with DFT pore size analysis. A main purpose of the present study was to demonstrate approaches to quantitatively analyze in situ SAXS/SANS coupling with physisorption. Further, our experiments clearly reveal for the first time a quantitative assessment of micropore volume and micropore size distribution based on advanced SANS/SAXS analysis. The obtained micropore volume and average micropore size agreed well with the NLDFT pore size analysis based on nitrogen adsorption. We have demonstrated suitable approaches to analyzing the scattering data with respect to the determination of such small, disordered pores in a complex pore hierarchy. It was possible to prove that the majority of the micropores is located between the larger pores, being attributable to the templating of the PEO chains of the block copolymer. The data obtained with dibromomethane will be used to validate sorption theory for gases other than nitrogen and rare gases. It was shown that for ordered mesopore systems the analysis of the intensity of the first Bragg reflection can provide the micropore volume, if the layer formation of the IL mesopores is taken into account correctly.

Furthermore, highly valuable information is obtained about the distribution of the three different pores (see Scheme 3): in essence, it can be concluded that the vast majority of the IL mesopores and micropores are located between the KLE mesopores. In addition, it can be also inferred that there are no extended domains of isolated KLE mesopores, which are not connected by the smaller pores. Thus, in the templating process no demixing takes place between the ionic liquid and the block copolymer. These insights thus help to further understand the templating mechanism itself and similar materials produced by other block copolymer templates ${ }^{36}$ or processed as film. ${ }^{37}$

(36) Yu, K.; Smarsly, B.; Brinker, C. J. Adv. Funct. Mater. 2003, 13, 47-52.

(37) Sel, O.; Sallard, S.; Brezesinski, T.; Rathouský, J.; Dunphy, D. R.; Collord, A.; Smarsly, B. M. Adv. Funct. Mater. 2007, 17, 3241-3250. 
Acknowledgment. Helmholtz-Zentrum Berlin (HZB) is gratefully acknowledged for financial support and the possibility to perform the in situ experiments. This work was supported by the German Research Foundation (DFG, SM199/7-1). Financial support from the Deutsches Elektronen-Synchrotron (DESY) and the European Community (reimbursement contract RII3-CT-2004-506008 (IA-SFS)) is gratefully acknowledged.

\section{Appendix: Pore fraction Calculation by Means of the Bragg Peak Analysis}

The different volume fractions $\tilde{\phi}$ were obtained by means of the eq 5 . In the case of the NLDFT method the single volumina were taken from Table 1. The application of the Bragg peak analysis considers the determination from the scattering pattern of the "partial" volume fractions $\phi$, i.e., the volume fractions of the pores (micropores and small IL mesopores) in the walls separating the KLE mesopores. Through the conversion of the eqs $4 a-4 c$, the single volumina values can be obtained as follows:

$$
\begin{aligned}
& V_{\mathrm{ILmesopores+micropores}}=\frac{\phi_{\mathrm{ILmesopores}+\text { micropores }} V_{\mathrm{SiO} 2}}{1-\phi_{\mathrm{ILmesopores}+\text { micropores }}} \\
& V_{\text {micropores }}=\left(1-\phi_{\mathrm{ILmesopores+micropores}}\right) \phi_{\text {micropores }} V_{\mathrm{SiO} 2}
\end{aligned}
$$

where $V_{\mathrm{SiO} 2}$ is obtained by the density of silica $\rho_{\mathrm{SiO} 2}$ and the mass of the analyzed sample $\mathrm{m}_{\mathrm{SiO} 2}=0.117 \mathrm{~g}$. The value of $V_{\text {ILmesopores }}$ is obtained by the difference between $V_{\text {ILmesopores+micropores }}$ and $V_{\text {micropores. }}$ The pore volume fraction $\tilde{\phi}$ can be calculated then by means of the eq 5 . Since $V_{\text {KLE pores }}$ cannot be determined with the Bragg peak analysis, the value obtained with the NLDFT is used also within this approach. The practical example of the $\tilde{\phi}_{\text {micropores }}$ calculation is shown here below.
From eq $8 b$

$$
\begin{gathered}
1-\phi_{\text {ILmesopores+micropores }}=\sqrt{\frac{27}{54}}=0.7 \\
\phi_{\text {ILmesopores+micropores }}=0.3 \\
1-\phi_{\text {micropores }}=\sqrt{\frac{27}{39}}=0.83 \\
\phi_{\text {micropores }}=0.17
\end{gathered}
$$

then from eq $\mathrm{A} 1$

$$
\begin{aligned}
V_{\text {ILmesopores+micropores }}=\frac{0.3 \times 0.053}{0.7} \mathrm{~cm}^{3}=0.023 \mathrm{~cm}^{3} \\
V_{\text {micropores }}=0.7 \times 0.17 \times 0.053 \mathrm{~cm}^{3}=0.0063 \mathrm{~cm}^{3} \\
V_{\text {ILmesopores }}=V_{\text {ILmesopores+micropores }}-V_{\text {micropores }} \\
=(0.023-0.0063) \mathrm{cm}^{3}=0.0167 \mathrm{~cm}^{3}
\end{aligned}
$$

From Table 1, the value of $V_{\mathrm{KLE}}=0.0151 \mathrm{~cm}^{3}$ is obtained, then with eq 5

$$
\widetilde{\phi}_{\text {micropores }}=\frac{0.0063 \mathrm{~cm}^{3}}{(0.0063+0.0167+0.0151+0.053) \mathrm{cm}^{3}}=0.07
$$

Supporting Information Available: Additional information as described in the text. This material is available free of charge via the Internet at http://pubs.acs.org. 